\title{
A multi-agent system to manage users and spaces in a Adaptive Environment System
}

\author{
Pedro Filipe Oliveira ${ }^{12}$, Paulo Novais ${ }^{1}$, and Paulo Matos ${ }^{2}$ \\ 1 Algoritmi Centre/University of Minho, Department of Informatics, Braga, Portugal, \\ 2 Institute Polytechnic of Bragança, Dep. of Informatics and Communications, \\ Bragança, Portugal
}

\begin{abstract}
This paper, deals with the actual problem of manage user preferences and local specifications on an IoT adaptive system, namely using a multi agent system to achieve a Smart Environment System. On a new era of interaction between persons and physical spaces, users want those spaces smartly adapt to their preferences in a transparent way. To achieve that, new approaches are needed. In this project we develop a multi agent system architecture with different layers to achieve a solution that entails all the proposed objectives.
\end{abstract}

Keywords: adaptive-system, AmI, multi-agent, IoT

\section{Introduction}

The Artificial Intelligence field continues with an exponential growth rate, especially in the different sectors applicability. Currently, multi-agent systems have been used to solve diverse situations.

Particularly in the AmI field, which is characterized by the creation of ubiquitous environments, interconnecting different technologies to perform common tasks of the user's daily life, autonomously, proactively and independently of the interaction of this [3].

This project proposes as well, a solution using a multi-agent system. Next, the problem will be detailed, as well as a solution proposal, which includes all the architecture developed, that later will be implemented and tested.

\section{Materials and Methods}

This work proposes an autonomous Smart Home model, controlled through cognitive agents, which get the final information to be applied by the actuators.

For do that, a house with five divisions was prototyped with different comfort features, namely temperature, luminosity, audio and video.

The considered parameters for performance evaluation are as follows:

- Number of agents used;

- Agent speed reasoning; 
- Information filtering;

- Environment perception time;

Figure 1, shows the scenario of an environment where it intends to develop this work. Explaining this figure, it can be seen the user who through its different devices (smartphone, wearable, and other compatible) communicates with the system, and for that can be used different technologies, like Near Field Communication (NFC) [7], Bluetooth Low Energy (BLE) [1] and Wi-Fi Direct [2]. Next, the system performs communication with the Cloud, to validate the information. And then the system will perform the management of the different components in the environment (climatization systems, security systems, other smart systems).

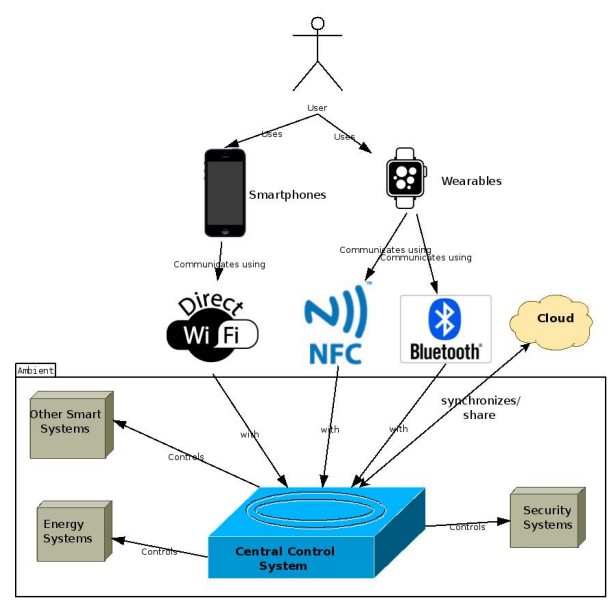

Fig. 1: Problem Statement

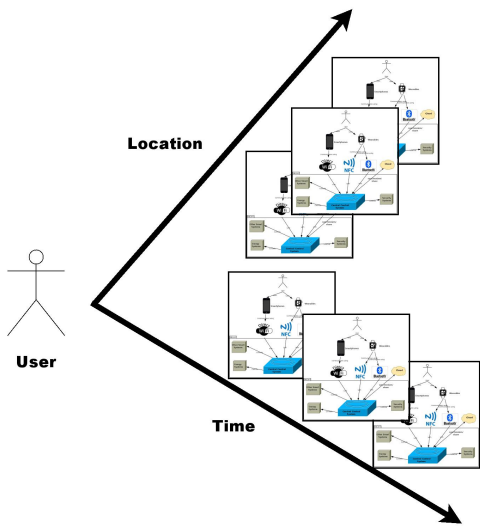

Fig. 2: Contextualization of Time/Environment Dimensions

To optimize the predictions of the solution proposed, an architecture for a multi-agent system was defined. The roles that each agent should represent, as well as the negotiation process to be taken, and the different scenarios in which this negotiation should take place and the way it should be processed were specified.

This work resulted in the complete specification of an architecture that supports the solution found, to solve the presented problem. It will now be implemented, tested and validated using real case studies, so as to gather statistical information to assess its effectiveness and performance in the context of application.

This work aims to give continuity and finalize the doctoral work presented in previous editions [4][5][6]. Thus the following diagram 3 exemplifies in a global way the architecture of the system where this work has been carried out. 


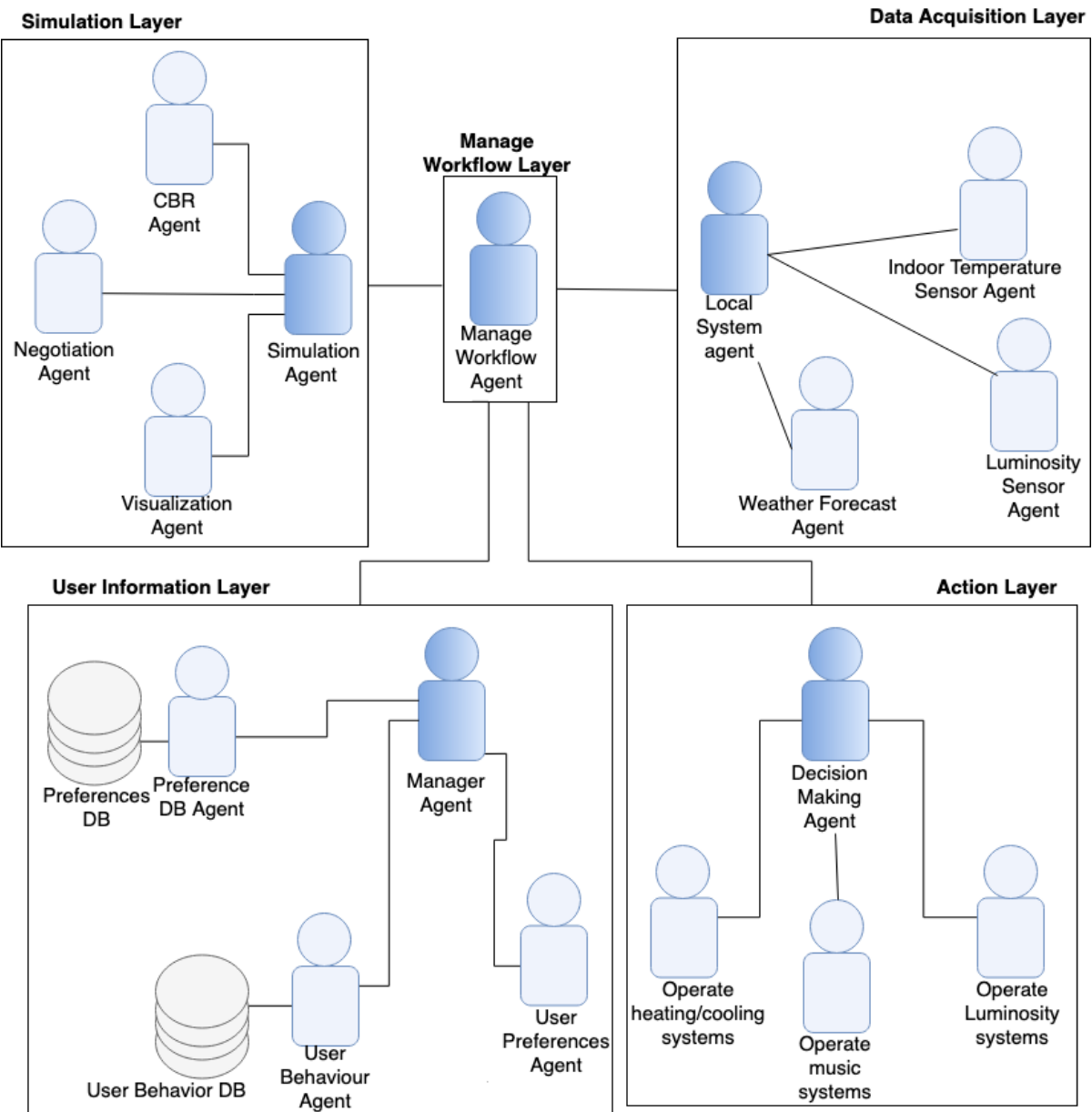

Fig. 3: Architecture of the multi-agent system

\section{$3 \quad$ Results}

Diagram 3 represent the architecture separation into different layers, to be easily identified the purpose of each, and agents containing it.

The layers description is as follows:

- Data acquisition layer, which will import the necessary information for the agents operation, namely information of interior and exterior temperature and light sensors.

- User layer, in this layer we will have an agent that will represent each user and his preferences that must be taken into account in the negotiation process. 
- Local System layer, here each local system will be represented by an agent, which contains all the information necessary to this location, either the referred to user preferences, or local/users security (maximum/minimum temperature, safety values for $\mathrm{CO} 2$, etc.).

- Simulation layer, in this layer will be the negotiation between the different agents involved, namely the management of conflicts between the different users and local systems. After the negotiation process ends we will have as result the values to apply in the place.

- Action layer, after the process is executed in the simulation layer, and the values to be applied are obtained. These values are used in this layer and sent to the actuators that will apply them in the different automation systems present the local.

\section{Discussion and Conclusions}

The agent system modeling is fully developed. The whole structure of data acquisition, to feed the information acquisition layer, is also completed. At this stage the agent layer is being developed and will be subsequently implemented for testing in the testing environment developed for this project.

\section{Acknowledgments}

This work has been supported by FCT - Fundação para a Ciência e Tecnologia within the Project Scope: UID/CEC/00319/2019.

\section{References}

1. Bluetooth, S.: Bluetooth core specification version 4.0. Specification of the Bluetooth System (2010)

2. Camps-Mur, D., Garcia-Saavedra, A., Serrano, P.: Device-to-device communications with wi-fi direct: overview and experimentation. Wireless Communications, IEEE 20(3), 96-104 (2013)

3. Chaouche, A.C., Seghrouchni, A.E.F., Ilié, J.M., Saïdouni, D.E.: A higher-order agent model with contextual planning management for ambient systems. In: Transactions on Computational Collective Intelligence XVI, pp. 146-169. Springer (2014)

4. Oliveira, P., Matos, P., Novais, P.: Behaviour analysis in smart spaces. In: 2016 Intl IEEE Conferences on Ubiquitous Intelligence \& Computing, Advanced and Trusted Computing, Scalable Computing and Communications, Cloud and Big Data Computing, Internet of People, and Smart World Congress (UIC/ATC/ScalCom/CBDCom/IoP/SmartWorld). pp. 880-887. IEEE (2016)

5. Oliveira, P., Novais, P., Matos, P.: Challenges in smart spaces: Aware of users, preferences, behaviours and habits. In: International Conference on Practical Applications of Agents and Multi-Agent Systems. pp. 268-271. Springer (2017)

6. Oliveira, P., Pedrosa, T., Novais, P., Matos, P.: Towards to secure an iot adaptive environment system. In: International Symposium on Distributed Computing and Artificial Intelligence. pp. 349-352. Springer (2018)

7. Want, R.: Near field communication. IEEE Pervasive Computing 0(3), 4-7 (2011) 